\title{
Development needs of nuclear data for fusion technology
}

\author{
U. Fischer ${ }^{1, a}$, P. Batistoni ${ }^{2}$, R. Forrest ${ }^{3}$, C. Konno $^{4}$, R.L. Perel ${ }^{5}$, K. Seidel ${ }^{6}$, and S.P. Simakov ${ }^{1}$ \\ 1 Association FZK-Euratom, Forschungszentrum Karlsruhe, Institut für Reaktorsicherheit, P.O. Box 3640, 76021 Karlsruhe, Germany \\ 2 Associazione ENEA-Euratom, ENEA Fusion Division, Via E. Fermi 27, 00044 Frascati, Italy \\ 3 Euratom/UKAEA Fusion Association, Culham Science Centre, Abingdon, Oxfordshire OX14 3DB, UK \\ 4 Japan Atomic Energy Agency, Tokai-mura, Naka-gun, Ibaraki-ken 319-1195, Japan \\ 5 Racah Institute of Physics, The Hebrew University of Jerusalem, 91904 Jerusalem, Israel \\ 6 Technische Universität Dresden, Institut für Kern- und Teilchenphysik, 01062 Dresden, Germany
}

\begin{abstract}
An overview is presented of the nuclear data required for nuclear design analyses of fusion technology focusing on ITER, the International Thermonuclear Experimental Reactor, and IFMIF, the International Fusion Materials Irradiation Facility. The status of the available data evaluations and libraries is reviewed with regard to the required materials/nuclides and data types and, in particular, with regard to their quality as compared to differential and integral experimental data. Future development needs are identified on this basis addressing nuclear data evaluations for neutron and photon transport simulations, cross section data for activation and transmutation calculations, and co-variance data for uncertainty analyses.
\end{abstract}

\section{Introduction}

The efforts in fusion technology aim at developing, on the long-term, power reactors which can contribute substantially to the supply of electricity. The roadmap leading to a commercial fusion power plant (FPP) considers the construction and operation of ITER, the International Thermonuclear Experimental Reactor [1], as an essential next step, followed by a demonstration (DEMO) power plant. The International Fusion Materials Irradiation Facility (IFMIF) [2] is assumed to be constructed and operated in parallel to ITER with the objective to test and qualify the materials for the high neutron fluences required for DEMO.

Neutronics and nuclear data have an essential role to play in this strategic approach. A well-qualified nuclear database and validated computational tools are required for reliable nuclear design calculations including uncertainty assessments. Quality assured neutronics and activation calculations, e.g., are mandatory for safety related nuclear analyses in the frame of the license process of the ITER device. Accordingly, the international efforts focus on the development and qualification of computational tools and nuclear data for quality assured design analyses. This includes the development of dedicated nuclear data libraries covering the materials and data types for the entire energy range of interest. In addition, substantial effort is devoted to integral experiments with the objective to provide the experimental data base for testing the nuclear data and validating the neutronics design calculations.

In the following, an overview is presented of the data required for nuclear design analyses in the context of the fusion technology strategy outlined above. The status of the available data evaluations and libraries is reviewed with regard to the required materials/nuclides and data types and, in particular, with regard to their quality as compared to differential and integral experimental data. On this basis, future development

${ }^{a}$ Presenting author, e-mail: ulrich.fischer@irs.fzk. de needs are identified addressing nuclear data evaluations for neutron and photon transport simulations, cross section data for activation and transmutation calculations, and co-variance data for uncertainty analyses.

\section{Nuclear data needs for fusion technology}

The nuclear design of any kind of fusion device relies on the results of particle transport calculations which provide the neutron/photon flux spectra and form the basis for the calculation of nuclear responses of interest when convoluted with related nuclear data. Appropriate and qualified computational simulations are required to ensure that the nuclear responses are reliable. These in turn require appropriate computational methods and tools along with well qualified nuclear data both for the transport simulation and the calculation of nuclear responses.

\subsection{Cross section data}

Neutron cross section data must be provided for all nuclides constituting the materials to be used in a fusion device, including the breeders, neutron multipliers, coolants, shielding, structure, magnets and insulators, with special emphasis on high-quality data around $14 \mathrm{MeV}$. A major feature is the importance of inelastic neutron reactions, which require the use of double-differential cross section data to properly describe the energy-angle distributions of emitted secondary neutrons. Photon production and interaction data must be included in the nuclear data libraries for use in coupled neutron-photon transport calculations. In addition, specific nuclear response data are required such as tritium production, kerma factors, gas production and radiation damage. Activation and transmutation cross section data must also be provided for the isotopes of all stable elements that may be present in the materials 
including impurities and tramp elements. Covariance data must be available to enable the assessment of uncertainty margins of the important nuclear responses.

For practical applications data evaluations must be complete, i.e., they must include all data types and nuclear reactions which are required for neutron and photon transport simulations as well as the calculation of relevant nuclear responses. For activation calculations, on the other hand, a full set of data for all potential target nuclides must be available. To allow the preparation of working libraries for use with state-ofthe-art transport and activation codes, complete data sets must be prepared in accordance with standard nuclear data format rules such as ENDF-6.

\subsection{Materials}

There is a variety of materials present in a complex fusion device such as ITER. This includes, e.g., materials of the Test Blanket Modules (TBM), the plasma facing components, the shield modules, the vacuum vessel, the super-conducting magnets, the bio shield as well as many other components.

TBM materials include $\mathrm{Be}$ (neutron multiplier), $\mathrm{Li}_{4} \mathrm{SiO}_{4}$, $\mathrm{Li}_{2} \mathrm{TiO}_{3}, \mathrm{~Pb}-\mathrm{Li}$ (breeder), the Eurofer reduced activation steel ( $\mathrm{Fe}, \mathrm{Cr}, \mathrm{W}, \mathrm{Ta}, \mathrm{V}, \mathrm{Mn}, \mathrm{C}$, and minor elements) [3] and the $\mathrm{He}$ coolant. Plasma facing components include $\mathrm{Be}, \mathrm{W}$, and $\mathrm{Cu} / \mathrm{Cu}$ alloy (CuCrZr, $\mathrm{CuA125}$ ). The shield modules and the vacuum vessel, e.g., is made of SS-316L (N) with $\mathrm{Fe}, \mathrm{Cr}, \mathrm{Ni}, \mathrm{Mo}, \mathrm{Mn}$, C, N as major constituents, SS 30467 (2w\% B) with $\mathrm{Fe}, \mathrm{Cr}$, $\mathrm{Ni}, \mathrm{Mo}, \mathrm{Mn}, \mathrm{B}, \mathrm{C}, \mathrm{N}, \mathrm{P}, \mathrm{S}$, and $\mathrm{H}_{2} \mathrm{O}$. The super-conducting magnet encompass the super-conducting strand with $\mathrm{Cu}, \mathrm{Nb}$, $\mathrm{Sn}, \mathrm{Ta}, \mathrm{Ti}$ and $\mathrm{Cr}$, the normal conducting $\mathrm{Cu}$ wire, the Helium coolant and Epoxy resin insulator with $\mathrm{Si}, \mathrm{O}, \mathrm{B}, \mathrm{Al}, \mathrm{H}$ and $\mathrm{C}$ as the main elements.

Since the near-term focus is on the TBM design, the elements $\mathrm{Be}, \mathrm{Pb}, \mathrm{Li}, \mathrm{Si}, \mathrm{O}, \mathrm{Fe}, \mathrm{Cr}, \mathrm{W}, \mathrm{Ta}, \mathrm{Cu}$, Ti are considered as high priority elements for which well qualified evaluated data sets are required.

The materials of highest importance for the IFMIF neutron source are the reduced activity ferritic-martensitic (RAFM) steels such as Eurofer that will be irradiated in the high flux test module (HFTM) of IFMIF up to a target fluence corresponding to $150 \mathrm{dpa}$. Other materials such as $\mathrm{SiC}, \mathrm{V} / \mathrm{V}-$ alloy, divertor materials (e.g., W), ceramic insulators or breeder/multiplier materials are considered for high fluence irradiations in IFMIF with lower priority. According to the elemental composition of Eurofer highest priority is assigned to the data of the main constituents $\mathrm{Fe}, \mathrm{Cr}, \mathrm{W}, \mathrm{V}, \mathrm{Mn}, \mathrm{C}$ and $\mathrm{Ta}$.

\subsection{Specific data needs for IFMIF}

The IFMIF neutron source uses d-Li reactions to produce neutrons for the high fluence irradiation of fusion power reactor candidate materials. To this end, a flowing liquid lithium target is bombarded by a high current deuteron beam accelerated up to $40 \mathrm{MeV}$ energy. The resultant neutron spectrum is fusionrelevant but includes a high-energy tail that extends up to $55 \mathrm{MeV}$.
Dedicated computational tools and data are required for IFMIF neutronics and activation calculations. These tools must be capable of simulating the transport of neutrons generated by $\operatorname{Li}(\mathrm{d}, \mathrm{xn})$ reactions and of the photons produced. Evaluated cross section data are thus required for the $\mathrm{d}+{ }^{6,7} \mathrm{Li}$ reaction system up to $40 \mathrm{MeV}$ deuteron energy.

Neutron cross section data must be provided over the whole neutron energy range up to $55 \mathrm{MeV}$. Such data must be evaluated for a variety of nuclides important for transport, activation and transmutation calculations. This includes also the deuteron-induced activation of a wide range of materials.

\section{Current status of nuclear data for fusion}

\subsection{Available fusion nuclear data libraries}

Various efforts have been conducted on the development of dedicated fusion nuclear data libraries, notably in the European Union (European Fusion File, EFF) and in Japan (JENDL-FF, Japanese Evaluated Nuclear Data Library - Fusion File), see, e.g., [4] for an overview. A major international effort was led by the IAEA/NDS when launching the FENDL (Fusion Evaluated Nuclear Data Library) project [5]. The most recent version of the data library, FENDL-2.1 [6], has been adopted as the current reference data library for ITER design applications.

In the frame of the EFF project of the European Fusion Programme, general purpose data evaluations have been prepared for ${ }^{9} \mathrm{Be},{ }^{28} \mathrm{Si},{ }^{46,47,48,49,50} \mathrm{Ti}$, ${ }^{\text {nat }} \mathrm{V},{ }^{52} \mathrm{Cr}$, ${ }^{56} \mathrm{Fe}$, and ${ }^{58,60} \mathrm{Ni}$ including covariance data up to $20 \mathrm{MeV}$. These EFF evaluations are included in the Joint Evaluated Fission and Fusion File JEFF-3.1 [7]. General purpose data evaluations up to $150 \mathrm{MeV}$ were performed for the stable tungsten isotopes ${ }^{182,183,184,186} \mathrm{~W}$ [8], ${ }^{181} \mathrm{Ta}$ [9] and, most recently ${ }^{55} \mathrm{Mn}$ [10]. These data files are complemented by covariance data and will be also integrated into the JEFF data library.

The JENDL Fusion File 99 [11] is a rather comprehensive data library for fusion applications. It comprises full data evaluations for 79 isotopes and 13 natural elements relevant to fusion applications. Most of the evaluations are based on up-dates of JENDL-3.1 data by including correlated energyangle distributions (double-differential cross sections) for the secondary particles.

Recently, substantial progress has been achieved in developing modern general purpose nuclear data libraries which also serve the specific needs of fusion applications. These include the latest versions of the data files from the European JEFF [7], the Japanese JENDL [12] and the American ENDF [13] projects.

\subsection{Specific data for IFIMF}

To provide the data for describing the source neutron generation in the lithium target of the IFMIF neutron source by means of the McDeLicious Monte Carlo code [14], a complete new evaluation of the $\mathrm{d}+{ }^{6,7} \mathrm{Li}$ interaction cross sections up to $50 \mathrm{MeV}$ deuteron energy has been performed. A new methodology was applied which takes into account compound 


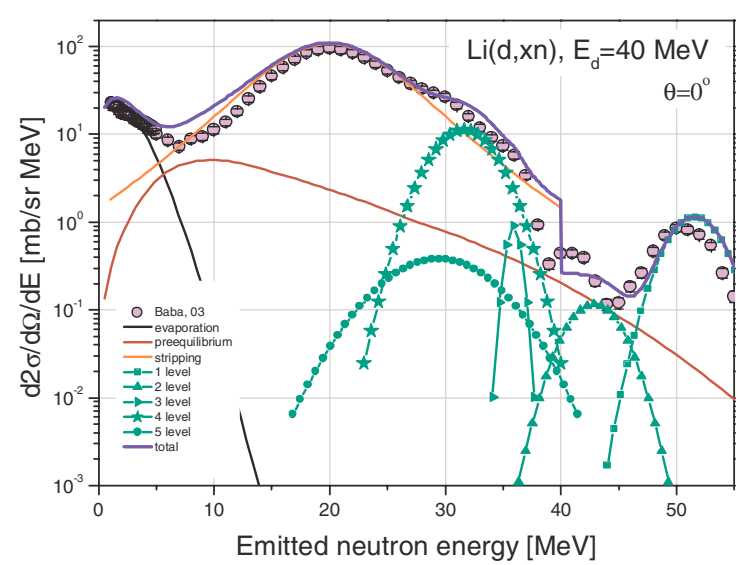

Fig. 1. Measured [16] and evaluated ${ }^{7} \mathrm{Li}(\mathrm{d}, \mathrm{xn})$ neutron emission cross section for $40 \mathrm{MeV}$ incident deuterons.

nucleus reactions, pre-equilibrium processes, stripping and direct interactions [15]. Figure 1 shows, as an example, the calculated double-differential neutron emission cross section and its breakdown into the different reaction components for $40 \mathrm{MeV}$ incident deuterons. It is revealed that the inclusion of direct interaction processes is essential for representing the structures of the emission cross section at high neutron energies. This is due to the fact that the direct reaction mechanism is dominant for the emission of neutrons with energies above the deuteron incidence energy.

The evaluation effort on general purpose neutron cross section data performed in the early stage of the IFMIF project focused on a few selected important nuclides up to $50 \mathrm{MeV}$ incident neutron energy. In the meantime, a variety of $150 \mathrm{MeV}$ cross section data evaluations are available from various sources including LANL [17] and NRG [18]. Most of these evaluations are contained in the general purpose data libraries of ENDF/B-VI, -VII and JEFF-3.1, respectively. New $150 \mathrm{MeV}$ data evaluations have been prepared for ${ }^{180,182-184,186} \mathrm{~W},{ }^{181} \mathrm{Ta}$ and ${ }^{55} \mathrm{Mn}$ in the frame of the EFF project. The most comprehensive data library for high energy applications, however, was elaborated by JAEA. The JENDL High Energy data file [12] extends up to $3 \mathrm{GeV}$ and comprises both neutron and proton induced cross section data. The first JENDL-HE release of 2004 comprised 66 nuclides. Another set of 37 nuclides has been evaluated in the meantime and is expected to be released in 2007. In addition, JAEA has generated a special PKA/Kerma data file JENDL/PK with PKA spectra for 29 elements with 78 isotopes for radiation damage calculations.

Thus a variety of intermediate and high energy data evaluations is available including most of the high priority nuclides for IFMIF.

\subsection{Activation cross section data}

A major evaluation effort on the production of a qualified activation data library for fusion inventory calculations has been conducted in the frame of the EU fusion technology programme. This has led to various versions of the European Activation File (EAF), with the current version EAF-2007 having an extended energy range up to $60 \mathrm{MeV}$ [19]. The EAF-2007 activation file thus satisfies the needs for activation calculations of the IFMIF neutron source and contains cross section data of 65,565 neutron-induced reactions on 816 targets. A previous EAF version, EAF-2003 with neutroninduced cross sections up to $20 \mathrm{MeV}$, has been adopted as the FENDL-2.1/A and JEFF3.1/A activation data libraries. EAF2007 includes also deuteron- and proton- induced activation data libraries. These libraries are mainly based on model calculations with the TALYS code [20] using global parameters. They were produced to enable activation calculations for the IFMIF accelerator components caused by beam losses.

A dedicated Intermediate Energy Activation File, IEAF2001, was previously developed for neutron-induced activation cross sections up to $150 \mathrm{MeV}$ [21]. An update of this data file is currently underway [22]. A complementary data file for proton induced reactions has already been elaborated [23].

\section{Benchmarking and validation}

Testing and validation are essential in the process of assuring the quality assurance of the nuclear data evaluations for application calculations. This is achieved through integral benchmark experiments and their computational analyses both for transport and activation experiments.

\subsection{Neutron transport benchmarks}

Numerous $14 \mathrm{MeV}$ neutron transport benchmark experiments on a variety of fusion-relevant materials have been performed in the past decade. For overviews on the achievements see, e.g., [24-27].

\section{Breeder mock-up experiment}

The recent focus of the experimental benchmark effort has been on neutronics TBM mock-ups. A first EU experiment of this kind has been performed on a TBM mock-up of the HCPB (Helium-Cooled Pebble Bed) breeder blanket at the Fascati Neutron Generator (FNG) to check and validate the capability of the neutronic codes and nuclear data to predict its tritium production capability [28]. The mock-up replicates the main characteristics of a breeder insert of the TBM-HCPB in ITER.

The tritium generated during irradiation in a series of $\mathrm{Li}_{2} \mathrm{CO}_{3}$ pellets located at different penetration depths in the mock-up was found to be underestimated in the calculations by 5 to $10 \%$ on average independent on the nuclear data used, see, e.g., figure 2 showing the $\mathrm{C} / \mathrm{E}$ comparison for the pellet stacks in the back of the assembly when calculated with EFF data. The results obtained indicate that design calculations for the tritium breeding ratio (TBR) of fusion power reactors employing a HCPB type breeder blanket are conservative. Thus an additional TBR margin is provided which allows to compensate for other potential uncertainties.

A Monte Carlo based sensitivity/uncertainty analysis was performed for the TBM mock-up neutronics experiment to 


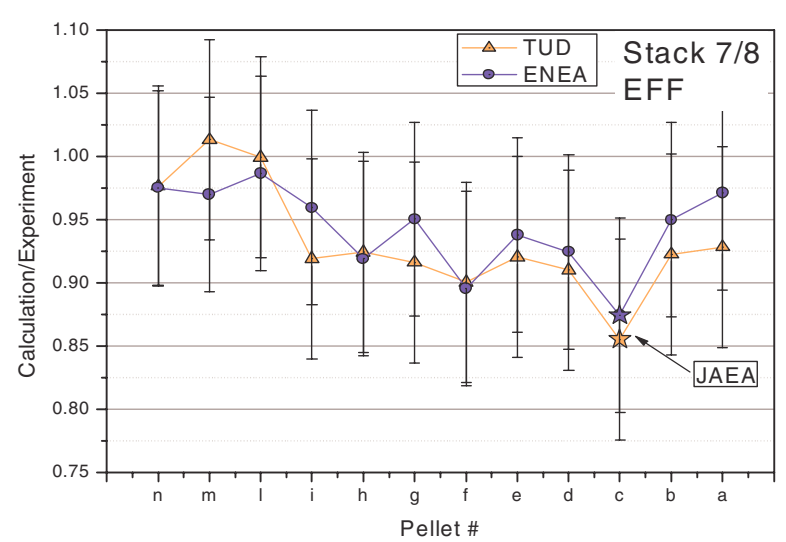

Fig. 2. Ratios of calculated (C) and experimental (E) tritium activities measured in pellets of the HCPB TBM mock-up [28].

assess the uncertainties of calculated tritium production rates and track them down to specific nuclides and reaction cross sections [29]. The track length estimator feature of the MCSEN code [30] was employed to this end. The dominant tritium production from ${ }^{6} \mathrm{Li}$, e.g., was shown to be mainly sensitive to the Be cross sections for the elastic scattering (1.7$2.1 \% / \%)$ and the $(\mathrm{n}, 2 \mathrm{n})$ reaction $(0.7 \% / \%)$. The uncertainties of the total Tritium Production Rate (TPR) due to uncertainties of the reaction cross sections are at $4 \%$ ( $2 \sigma$ confidence level) and are dominated by the ${ }^{9} \mathrm{Be}$ uncertainties. The total TPR uncertainties including the data uncertainties, the statistical uncertainties of the Monte Carlo calculation and the experimental uncertainties are in the order of 8 to $10 \%(2 \sigma)$.

\section{$\mathrm{Be}, \mathrm{Ti}, \mathrm{V}, \mathrm{Pb}, \mathrm{W}$ and Ta benchmarking}

A series of benchmark analyses has been recently performed on the cross sections of $\mathrm{Be}, \mathrm{Ti}, \mathrm{V}, \mathrm{Pb}, \mathrm{W}$ and $\mathrm{Ta}[31,32]$ with the objective to check and validate the related recent data evaluations for neutron transport calculations. Available integral benchmark experiments have been analysed to this end on the basis of Monte Carlo calculations with the MCNP Monte Carlo code using mainly EFF-3/JEFF-3.1 and FENDL2.0/2.1 data evaluations. The analyses also comprised checks of processed cross section data including comparisons to experimental data such as $14 \mathrm{MeV}$ neutron emission cross sections. Detailed results are given in various internal reports $[31,32]$ and will be included in the forthcoming JEFF-3.1 benchmark report. In the following, the main results are briefly summarized.

The new JEFF-3.1 lead evaluation was shown to underestimate measured neutron multiplication factors and neutron leakage spectra as well as the photon emission and leakage spectra. It was concluded that the JEFF-3.1 lead evaluation needs to be updated for fusion technology applications.

The new EFF-3 W data evaluation gives a good reproduction of the photon spectra measured at four different detector positions $(5,15,25$ and $35 \mathrm{~cm}$ depth) in a $49 \mathrm{~cm}$ thick tungsten assembly. Underestimation of the neutron flux spectra were obtained, however, in the high energy $(E>$ $12 \mathrm{MeV})$ and low energy (1-5 MeV) range. This results in an

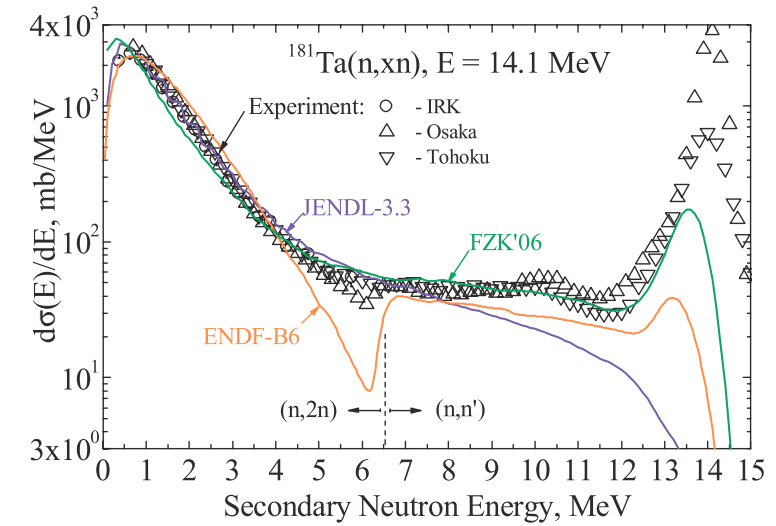

Fig. 3. Comparison of measured and calculated $\mathrm{Ta}\left(\mathrm{n}, \mathrm{xn}^{\prime}\right)$ neutron emission spectra at $14.1 \mathrm{MeV}$ incident neutron energy.

underestimation of the fast $(E>0.1 \mathrm{MeV})$ neutron flux up to $30 \%$ at the deepest detector location in the $\mathrm{W}$ assembly. The neutron emission spectrum, on the other hand, is well reproduced by the new $\mathrm{W}$ data evaluation. The same is true for the angular distributions of the secondary neutrons except for the angle of $30^{\circ}$.

Benchmark analyses on Ta have been conducted for the new ${ }^{181}$ Ta data evaluation provided by FZK for the EFF project [9]. Good agreement was obtained for the neutron leakage spectra measured in available neutron transmission experiments. Satisfactory agreement was also found for the neutron emission spectra while the other available ${ }^{181}$ Ta evaluations (ENDF/B-VI and JENDL-3.3) show severe discrepancies, see figure 3. Thus the new Ta data evaluation has been shown as a clear improvement over the deficient ENDF/B-VI and JENDL-3.3 Ta data evaluations.

The JEFF-3.1 vanadium evaluation, which originates from ENDF/B-VI, was shown to better reproduce the measurements of the neutron flux spectra than the other data evaluations while the photon flux spectra are still significantly overestimated.

\section{FENDL-2.1 benchmarking}

Extensive benchmark tests have been performed to check the quality of the new ITER reference nuclear data library FENDL-2.1. A series of available benchmark experiments has been re-analysed to this end using FENDL-2.1 data, see, e.g., [33] for the experiments performed at FNG in the framework of the EU fusion technology programme. Corresponding FENDL-2.1 benchmark tests have been performed by JAEA for the benchmark experiments conducted previously at the FNS [34]. As a general trend it was observed that the results obtained with FENDL-2.1 and -2.0 and those of other up-to date data evaluations agree to a large extent. For some cases, such as W, a clear improvement was obtained with FENDL2.1 data.

\subsection{D-Li neutron yield data}

A series of benchmark calculations were performed with the McDeLicious code to test the new $2005 \mathrm{Li}(\mathrm{d}, \mathrm{xn})$ data 


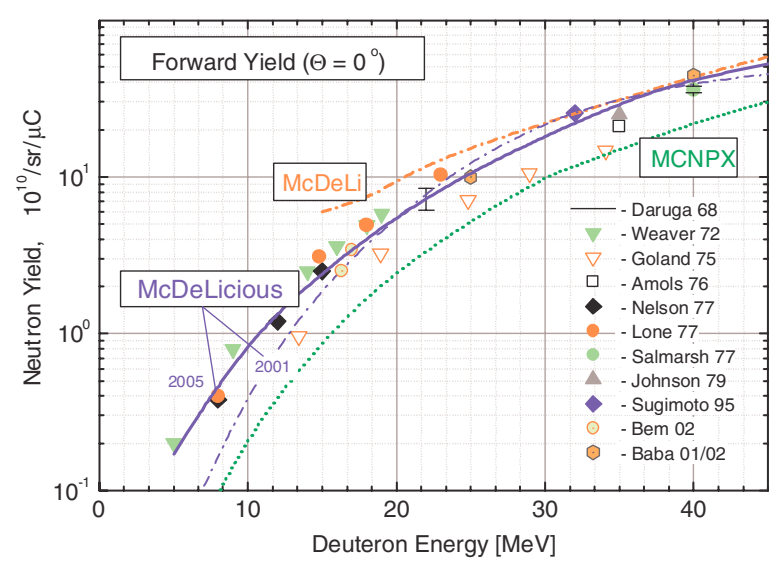

Fig. 4. Measured and calculated thick lithium target forward neutron yields.

evaluation against experimental thick lithium target neutron yields. The comparison of measured and calculated forward neutron yields, figure 4 , shows that McDeLicious with the new d-Li cross section data is well able to reproduce the experimental results over the entire deuteron energy range from threshold up to $40 \mathrm{MeV}$. The approaches of McDeLi [35], the predecessor to McDeLicious, and the MCNPX code [36] using the ISABEL intra-nuclear cascade model, give significant worse agreement with the experimental neutron yields.

The comparison of McDeLicious calculations for neutron angular differential yields using the new d-Li evaluation showed that the angular dependence can be satisfactorily predicted over the whole range of measured deuteron energies and secondary neutron angles [15]. The other two approaches show again a worse reproduction of the experimental data set, especially below $20 \mathrm{MeV}$. The comparison of McDeLicious calculations with measured double-differential thick target neutron yields demonstrates also significant improvements achieved with the updated d-Li evaluation.

\subsection{Activation analyses}

A major experimental effort has been recently conducted on the validation of fusion relevant activation cross section data [37]. Various fusion candidate materials such as the steels SS316, MANET, F82H and Eurofer, different vanadium alloys, pure elements ( $\mathrm{Al}, \mathrm{V}, \mathrm{Ni}, \mathrm{Cu}, \mathrm{Cr}, \mathrm{Fe}, \mathrm{Hf}, \mathrm{Nb}, \mathrm{Mo}, \mathrm{La}$, $\mathrm{Sn}, \mathrm{Y}, \mathrm{Pb}, \mathrm{W}, \mathrm{Ta}$ ), $\mathrm{CuCrZr}, \mathrm{SiC}$ and the $\mathrm{Li}_{4} \mathrm{SiO}_{4}$ breeder ceramics have been irradiated at $14 \mathrm{MeV}$ neutron generators and analyzed using the FISPACT code with EAF data. The results were used to update and improve the EAF cross section data for the current version EAF-2007 [19]. A recent example of a validated reaction is given in figure 5 for the reaction ${ }^{182} \mathrm{~W}(\mathrm{n}, \mathrm{p}){ }^{182}$ Ta reaction showing the $\mathrm{C} / \mathrm{E}$ ratio of the library to experimental cross sections averaged in different neutron flux spectra. The band indicates the uncertainty of the EAF-2007 cross section data.

Activation cross section data relevant for IFMIF were tested against irradiation experiments in a white neutron spectrum extending up to $32 \mathrm{MeV}$. This spectrum was produced at

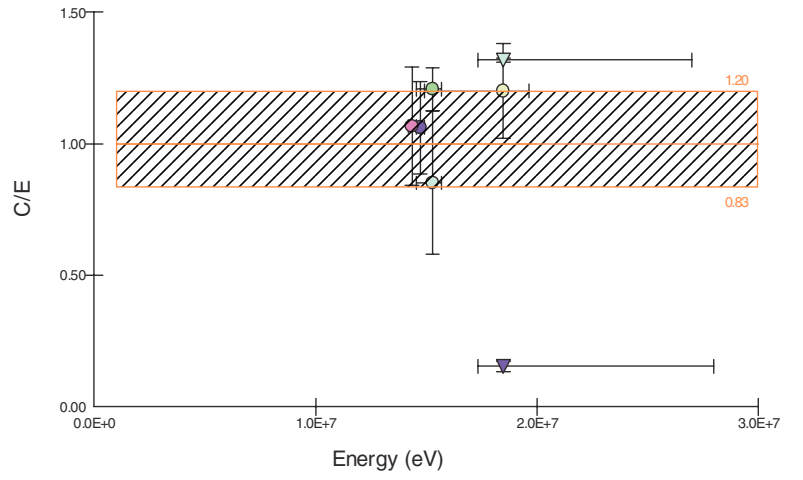

Fig. 5. Integral data for the reaction ${ }^{182} \mathrm{~W}(\mathrm{n}, \mathrm{p})^{182} \mathrm{Ta}$ using EAF-2007 cross section data.

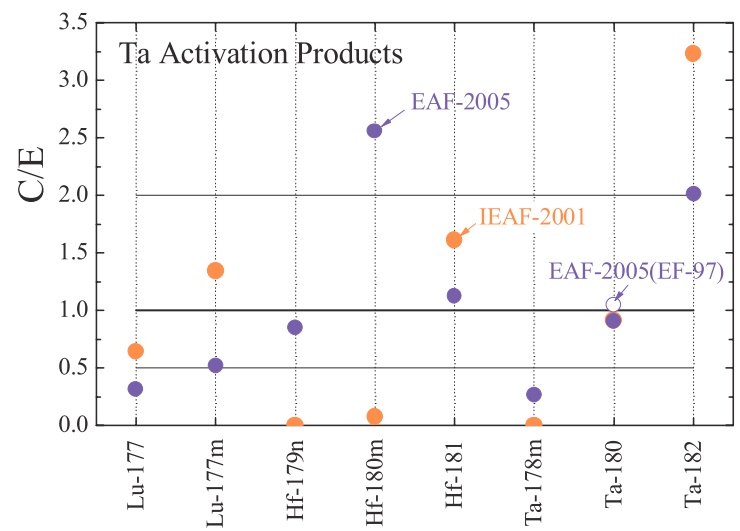

Fig. 6. Comparison of calculated and measured radio-activities induced by irradiating Ta samples in an IFMIF-like white neutron field.

the cyclotron of the Nuclear Physics Institute of Rez, Czech Republic, by bombarding a heavy water target with a $37 \mathrm{MeV}$ proton beam [38]. Recent irradiation experiments were performed on samples of Eurofer [39], W, Ta and Cr [40]. Results of the activation analyses for Ta are shown in figure 6 in terms of $\mathrm{C} / \mathrm{E}$ ratios of the resulting $\gamma$-activities. It is noted that the yields of ${ }^{180} \mathrm{Ta},{ }^{179 n} \mathrm{Hf}$ and ${ }^{181} \mathrm{Hf}$, can be well reproduced by EAF-2005 data while there are large discrepancies for other activation products of Ta. The analyses of the relevant reaction cross sections revealed large discrepancies between EAF-2005 and IEAF-2001 data and missing experimental data above $20 \mathrm{MeV}$.

\section{Further development needs}

Significant progress has been achieved over the past two decades in developing qualified nuclear data for fusion technology. Further development needs follow from the requirements of the long-term fusion programme with focus on the next step facilities ITER and IFMIF.

A complete general-purpose nuclear data library needs to be developed for IFMIF neutronics calculations. A variety of intermediate energy data evaluations is already available from JEFF, ENDF/B and the JENDL/HE data library including many of the IFMIF high priority nuclides. These evaluations need to be qualified and complemented for the remaining isotopes. 
Activation data libraries have been developed for neutron, proton and deuteron induced reactions according to the IFMIF needs. They are based, however, to a large extent on nuclear model calculations, in particular with regard to the high energy range. Thus there is a strong need for measuring IFMIF relevant activation cross data in the neutron energy range 20-50 MeV including elements such as $\mathrm{Cr}$, Co, V, W, Ta, $\mathrm{Pb}, \mathrm{Bi}, \mathrm{Tm}, \mathrm{Fe}, \mathrm{Au}, \mathrm{Mn}$, and others. Some of these data are required for applying the dosimetry foil activation technique to neutron flux monitoring [41]. The primary focus for deuteron induced cross sections is on the materials $\mathrm{Cu}, \mathrm{Al}$ and $\mathrm{Nb}$. Analogous data are required for proton induced activation reactions when operating the IFMIF prototype accelerator. This will include materials such as Ta, W, Pt, $\mathrm{Au}, \mathrm{Pb}$ that may be used for the proton beam dump.

Special attention must be paid to the gas production cross section data of the major IFMIF irradiation materials such as $\mathrm{Fe}, \mathrm{Cr}, \mathrm{Mn}, \mathrm{W}$ and Ta, and others. Recent measurements of the helium and hydrogen production cross sections by Haight et al. [42,43] have shown large disagreements between the experimental data and the nuclear model calculations in the neutron energy range 20 to $100 \mathrm{MeV}$.

With regard to ITER neutronics, the most urgent need is to continue with the improvement of data evaluations required for the neutronics analyses of the Test Blanket Modules (TBM) such as $\mathrm{Li}, \mathrm{Be}, \mathrm{Pb}$ and $\mathrm{O}$. A major challenge in this field is to provide the covariance data required for assessing the uncertainties of the nuclear responses in the TBM. This requires revisiting older evaluations without any uncertainty information in the data files. Covariance data can be obtained either from analyses of experimental data or, in cases where the experimental data are limited, by means of simulation calculations following new theoretical approaches.

This work, supported by the European Communities under the contract of Association between EURATOM and Forschungszentrum Karlsruhe was carried out within the framework of the European Fusion Development Agreement. The views and opinions expressed therein do not necessarily reflect those of the European Commission.

\section{References}

1. N. Holtkamp, in Proc. $24^{\text {th }}$ Symp. on Fusion Technology, Warsaw, Poland, Sept. 11-15, 2006 (to be published).

2. H. Matsui, in Proc. $21^{\text {st }}$ IAEA Fusion Energy Conference, Chengdu, China, 16-21 Oct. 2006.

3. R. Lindau, M. Schirra, Fus. Eng. Des. 58-59, 781-785.

4. U. Fischer et al., in Int. Conf. on Nucl. Data for Sci. and Technol., Santa Fe, 26. Sept.-1 Oct. 2004, USA, AIP Conf. Proc. 769, (2005), p. 1478.

5. D. Muir, S. Ganesan, in Proc. Int. Conf. Nucl. Dat. Sci. Technol., Jülich, May 13-17, 1991.
6. D.L. Aldama, A. Trkov, FENDL-2.1: Report INDC(NDS)-467, December 2004.

7. A.J. Koning et al. (these proceedings).

8. P. Pereslavtsev, U. Fischer, in Int. Conf. on Nucl. Data for Sci. and Technol., Santa Fe, 26. Sept.-1 Oct. 2004, USA (AIP Conf. Proc. 769, 2005), p. 215.

9. P. Pereslavtsev, U. Fischer, Nucl. Instrum. Meth. Phys. Res. B 248, 225 (2006)

10. P. Pereslavtsev, U. Fischer (these proceedings).

11. S. Shibata et al., J. Nucl. Sci. Technol. 39, 187 (2002).

12. K. Shibata et al. (these proceedings).

13. P. Oblozinsky et al. (these proceedings).

14. S. P. Simakov et al., J. Nucl. Mat. 307-311, 1710 (2002).

15. U. Fischer et al., in 12th Int. Conf. on Fusion Reactor Materials, Dec. 4-9, 2005, Santa Barbara, USA.

16. M. Hagiwara, M. Baba et al., in JAERI Nuclear Data Symposium, December 2002.

17. M.B. Chadwick, P.G. Young et al., Nucl. Sci. Eng. 131, 293 (1999).

18. A.J. Koning, M.C. Duijvestijn (these proceedings).

19. R.A. Forrest, J. Kopecky (these proceedings).

20. A. Koning, S. Hilaire, M. Duijvestijn (these proceedings).

21. U. Fischer et al., Intermediate Energy Activation File IEAF2001, NEA-1656/01 (2001), DLC-217/IEAF-2001 (2002).

22. P. Pereslavtsev, U. Fischer, S. Sudar (these proceedings).

23. A. Konobeyev et al. (these proceedings).

24. U. Fischer et al., Fus. Eng. Des. 51-52, 663 (2000).

25. I. Murata, A. Takahashi, J. Nucl. Sci. Technol. Suppl. 2, 1112 (2002).

26. P. Batistoni et al., Fus. Eng. Des. 81, 1169 (2006).

27. S. Sato et al., Fus. Eng. Des. 81, 1182 (2006).

28. P. Batistoni et al., in $24^{\text {th }}$ Symp. on Fusion Technology, Warsaw, Poland, Sept. 11-15, 2006.

29. D. Leichtle et al., in $24^{\text {th }}$ Symp. on Fusion Technology, Warsaw, Poland, Sept. 11-15, 2006.

30. R.L. Perel, J.J. Wagschal, Y. Yevein, Nucl. Sci. Eng. 124, 197 (1996).

31. U. Fischer, D. Leichtle, S. Simakov, Final report on the EFDA task TW5-TTMN-001, Deliverable 6, FZK, January 2006.

32. U. Fischer, D. Leichtle, S. Simakov, Final report on the EFDA task TW6-TTMN-001, Deliverable 5, FZK, March 2007.

33. P. Batistoni et al. (these proceedings).

34. C. Konno (2007) (private communication).

35. P.P.H. Wilson, Report FZKA 6218, January 1999.

36. L.S. Waters (ed.), MCNPX User's Manual Version 2.1.5, http://mcnpx.lanl.gov.

37. R.A. Forrest et al., Culham report UKAEA FUS 526 (2006).

38. P. Bem et al. (these proceedings).

39. P. Bem et al., Fus. Eng. Des. 75-79, 829 (2005).

40. M. Honusek et al. (these proceedings).

41. S. Simakov et al., in Proc. $24^{\text {th }}$ Symp. on Fusion Technology, Warsaw, Poland, Sept. 11-15, 2006 (to be published).

42. R.C. Haight, Los Alamos National Laboratory Report LA-UR04-4010, 2004.

43. R.C. Haight (these proceedings). 